$\mathrm{E}$

EVALUAR
2018, Vol. 18, No 1.

ISSN 1667-4545

Recuperado de https://revistas.unc.edu.ar/index.php/revaluar

Laboratorio de Evaluación Psicológica y Educativa

Facultad de Psicología - Universidad Nacional de Córdoba

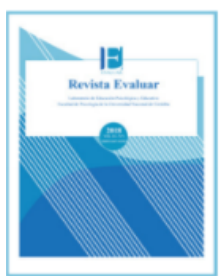

\title{
Desarrollo y Validación de un instrumento para medir Estrategias de Afrontamiento Religioso
}

\section{Development and Validation of an Instrument for the Measurement of Religious Coping Strategies}

\author{
Juan Aníbal González-Rivera * 1, Orlando M. Pagán-Torres ${ }^{2}$ \\ 1 - Universidad Carlos Albizu, San Juan, Puerto Rico. \\ 2- Ponce Health Sciences University, Ponce, Puerto Rico.
}

Recibido: 20/12/2017 Revisado: 10/01/2018 Aceptado: 11/01/2018

\section{Resumen}

El presente estudio analiza las propiedades psicométricas del Inventario de Estrategias de Afrontamiento Religioso (IEAR) en una muestra de adultos puertorriqueños. El afrontamiento religioso se define como las diferentes formas de entender y manejar los eventos negativos de la vida que guardan relación con lo sagrado. Un total de 350 puertorriqueños participaron en este estudio de carácter exploratorio y psicométrico. Los resultados confirmaron que la escala posee una estructura de dos factores. Estos factores fueron identificados como estrategias internas y estrategias externas. Un total de 12 reactivos cumplieron con los criterios de discriminación y cargas factoriales apropiadas (seis reactivos por factor). El índice de confiabilidad alfa de Cronbach de la escala fue .95. Estos resultados sugieren que el IEAR tiene el potencial para medir este constructo en adultos puertorriqueños. Además, la IEAR permitirá el avance de nuevas investigaciones sobre el afrontamiento religioso en Puerto Rico y América Latina.

Palabras Clave: afrontamiento religioso, afrontamiento, religiosidad, propiedades psicométricas, validación

\begin{abstract}
This article examines the psychometric properties of the Religious Coping Strategies Inventory (IEAR) in a sample of Puerto Rican adults. Religious coping methods are ways of understanding and dealing with negative life events that are related to the sacred. A total of 350 Puerto Ricans participated in this exploratory and psychometric study. The results indicated that the scale has a two-factor structure. These factors were denominated internal strategies and external strategies. A total of 12 items complied with the criteria of discrimination and presented appropriate factorial loads (six items per factor). The reliability index of the scale was of .95 (Cronbach's alpha). These results suggest that the IEAR has the potential to measure this construct among Puerto Rican adults. Likewise, the IEAR will advance further research of religious coping in Puerto Rico and Latin America.
\end{abstract}

Keywords: religious coping, coping, religiosity, psychometric properties, validation

*Correspondencia a: Dr. Juan A. González-Rivera, 500 West Main Suite 215, Bayamón, Puerto Rico, 00961. Tel.: 011787 315 6034. dr.juananibalgonzalez@outlook.com Cómo citar este artículo: González-Rivera, J. A., \& Pagán-Torres, O. M. (2018). Desarrollo y validación de un instrumento para medir Estrategias de Afrontamiento Religioso. 


\section{Introducción}

Las investigaciones basadas en la evidencia sobre religión, espiritualidad y salud, documentadas a través de revisiones narrativas y sistemáticas de literatura, han revelado las múltiples formas como la religión y la espiritualidad pueden impactar, tanto positiva como negativamente, en la salud mental y física de los individuos (Bonelli \& Koenig, 2013; Green \& Elliott, 2010; Koenig, 2008, 2012, 2015). Dichos hallazgos han quebrado los supuestos que en principio establecieron una dicotomía entre la religión y la psicología (Scharrón del Rio, 2010) y han llevado a los profesionales de la salud a concebir una integración entre la religión y la salud (González-Rivera, 2015; Koenig, 2008; Pagán-Torres, Reyes-Estrada, \& Cumba-Avilés, 2017).

Los hallazgos positivos documentados entre la religión, la espiritualidad y la salud han cosechado el desarrollo de un sinnúmero de técnicas religiosas y espirituales de intervención que han sido integradas en los procesos psicoterapéuticos. Esta iniciativa ha surgido con el propósito de ofrecer servicios de calidad a las personas que poseen necesidades religiosas y espirituales (Pagán-Torres et al., 2017) o algún problema de índole religiosa o espiritual (Asociación Americana de Psiquiatría, 2013). Algunas de las técnicas religiosas y espirituales que pueden integrarse en la psicoterapia son: la oración, el afrontamiento religioso y espiritual, la práctica del yoga, la meditación y la lectura de textos sagrados (González-Rivera, 2015; Richards \& Bergin, 2014). Sin embargo, la literatura empírica actual indica que el desarrollo de instrumentos que midan técnicas o estrategias religiosas y espirituales ha sido limitado.

Teniendo esto en consideración, este proyecto tuvo como objetivo principal desarrollar y validar un instrumento que mida, específicamente, las estrategias de afrontamiento religioso utilizadas por las personas para manejar eventos adversos según el marco teórico de Pargament (1997). A estos efectos se diseñó un estudio para evaluar si el instrumento poseía propiedades psicométricas adecuadas para ser utilizado en los contextos clínico e investigativo, y para comprobar si la distribución factorial del instrumento se adecuaba a las dimensiones teóricas propuestas para su desarrollo. Con este propósito, analizamos las propiedades psicométricas del instrumento con técnicas avanzadas y examinamos su estructura factorial. Esto permitirá ofrecer a la comunidad científica un instrumento válido y confiable que contribuya al avance del estudio de la psicología de la religión y la espiritualidad en América Latina.

\section{Religión, afrontamiento y afrontamiento religioso}

Antes de exponer la definición conceptual de afrontamiento religioso, se considera relevante definir los constructos religión, afrontamiento y afrontamiento religioso. La religión es definida como un sistema de creencias, prácticas espirituales o ambas organizadas en torno a la adoración de una deidad o deidades todopoderosas, e incluye comportamientos como plegarias, rezos, meditación y participación en rituales públicos (Asociación Americana de Psicología, 2010). El afrontamiento es definido como los esfuerzos cognitivos y conductuales realizados para dominar, tolerar o reducir las demandas externas e internas que son evaluadas como excedentes de los recursos del individuo (Folkman \& Lazarus, 1980). Por su parte, Pargament (1997) define afrontamiento como "la búsqueda de significado en tiempos de estrés" 
(p. 90). Por último, el afrontamiento religioso es definido como las diferentes formas, relacionadas con lo sagrado, de entender y manejar los eventos negativos de la vida (Pargament \& Raiya, 2007).

\section{Afrontamiento religioso}

Originalmente, el constructo afrontamiento religioso fue desarrollado por Pargament (1997), quien propone que la religión es una de las maneras mediante las cuales los individuos pueden afrontar sus situaciones de vida mediante la fortaleza interna que emerge de sus creencias y prácticas religiosas. Además, este autor sugiere que las personas con niveles más altos de afrontamiento religioso serán quienes más se beneficien de este método (Pargament, Koenig, Tarakeshwar, \& Hahn, 2001). De acuerdo a Pargament, Koenig y Pérez (2000) la religión brinda significado y propósito a la vida diaria de las personas y a los momentos de crisis. Debido a ello, estos autores conceptualizan el constructo afrontamiento religioso en cinco funciones claves: significado, control, comodidad, intimidad y transformación en la vida.

Por otro lado, Pargament, Smith, Koenig y Pérez (1998) subdividen los métodos de afrontamiento religioso en dos clasificaciones principales: afrontamiento religioso positivo y afrontamiento religioso negativo. El afrontamiento religioso positivo consiste en estrategias de colaboración, búsqueda de apoyo espiritual, conexión espiritual, purificación religiosa, búsqueda de ayuda de miembros del clero, ayuda religiosa y perdón religioso. Por otra parte, el afrontamiento religioso negativo consiste en estrategias asociadas a revalorizaciones religiosas punitivas, reconsideraciones religiosas demoníacas, reevaluaciones de los poderes de Dios, descontento espiritual, afrontamiento religioso autodirigido y descontento religioso interpersonal. Dichos métodos de afrontamiento están directamente asociados con niveles bajos de salud mental (Ano \& Vasconcelles, 2005).

Por otra parte, Lazarus y Folkman (1986) definen el afrontamiento como aquellos esfuerzos cognitivos y conductuales constantemente cambiantes que se desarrollan para manejar las demandas específicas, externas o internas, que son evaluadas como excedentes o desbordantes de los recursos del individuo. Desde esta perspectiva, desarrollamos nuestro marco teórico de afrontamiento religioso paralelo al modelo de Lazarus y Folkman (1986) considerando estrategias de afrontamiento religioso internas (cognitivas) y externas (conductuales). Las estrategias de afrontamiento religioso internas se caracterizan por la confianza y búsqueda de consuelo en un dios, la oración personal, seguridad en las creencias religiosas y la búsqueda de sentido religioso al sufrimiento. Al igual que Lazarus y Folkman (1986), consideramos que las estrategias internas facilitan el control de emociones en momentos de crisis.

Por su parte, las estrategias de afrontamiento religioso externas están vinculadas con actividades en las que el individuo busca fuentes de apoyo provenientes de algo externo a sí mismo como, por ejemplo, buscar apoyo en una comunidad de fe, asistir a la iglesia, leer libros religiosos, escuchar música sacra y recibir consejería/ dirección espiritual. Consiguientemente, nuestra visión teórica de estrategias internas y externas guarda consistencia con los planteamientos del modelo cognitivo-conductual de Beck (2011). Según este modelo, para manejar aquellos eventos o situaciones que puedan afectar la salud mental se debe identificar qué pensamientos y acciones influyen en el estado de ánimo y así tener un mejor manejo de los mismos. 
Instrumentos para medir afrontamiento $y$ afrontamiento religioso

En sus inicios, los estudios empíricos enmarcados dentro del tópico de la religión y la espiritualidad no contaban con instrumentos psicométricamente aptos para medir el constructo de afrontamiento religioso. Esto se hace notable al examinar los instrumentos de afrontamiento más utilizados en las investigaciones contemporáneas. Por ejemplo, el COPE Inventory (Carver, Scheier, \& Weintraub, 1989) sólo contiene cuatro reactivos asociados con el afrontamiento religioso: 7 (Pongo mi confianza en Dios), 18 (Busco la ayuda de Dios), 48 (Intento encontrar comodidad con mi religión) y 60 (Oro más de lo usual). Sucede lo mismo con el Ways of Coping Questionnaire (Folkman \& Lazarus, 1988), de sus 66 reactivos, únicamente dos son de contenido religioso: "Oré" y "Encontré una nueva fe". Por otro lado, el Coping Strategy Questionnaire (Rosenstiel \& Keefe, 1983) contiene en su versión original 50 reactivos. De estos, cinco reactivos conforman la subescala de Oración/Esperanza: (1) Ruego a Dios que no dure demasiado, (2) Oro para que el dolor se detenga, (3) Sé que algún día alguien estará aquí para ayudarme y desaparecerá por un tiempo, (4) Confio en mi fe en Dios y (5) Tengo fe en los médicos que algún día habrá una cura para mi dolor. Con relación al Coping Inventory for Stressful Situations desarrollado por Endler y Parker (1999), encontramos que en ninguna de sus dimensiones los autores consideran el afrontamiento religioso.

Ante la necesidad de un instrumento que permitiera profundizar en el estudio de las estrategias de afrontamiento religioso, Pargament et al. (2000) desarrollaron el primer instrumento válido para medir afrontamiento religioso: The Religious Cope (RCOPE). Esta escala, en su forma original, posee 105 reactivos distribuidos en 21 subescalas. Según lo informado en el estudio, las estimaciones de confiabilidad del instrumento fueron altas. El RCOPE obtuvo una consistencia interna de .80 o más para todas las subescalas con excepción de dos dimensiones: Marcando los límites religiosos con .78 y La reevaluación del poder de Dios con .61. Más adelante, Pargament, Feuille y Burdzy (2011) desarrollaron una versión breve con 14 reactivos (Brief COPE). Asimismo, se desarrolló el Children's Religious Coping Scale (CRC), mediante un estudio cualitativo sobre estrategias de afrontamiento religioso en niños (Pendleton, Cavalli, Pargament, \& Nasr, 2002).

Partiendo de lo antes mencionado, la literatura documenta un esfuerzo en el contexto latinoamericano en relación con el desarrollo y validación de instrumentos psicométricamente aptos para medir el afrontamiento religioso. Panzini y Bandeira (2005) elaboraron la Spiritual/ Religious Coping Scale (SRCOPE Scale), validada para la población brasileña. Esta escala contiene un total de 66 reactivos distribuidos en ocho factores y obtuvo un índice de consistencia interna alfa de Cronbach de .97 para la escala total.

Necesidad de un Instrumento de Estrategias de Afrontamiento Religioso en Puerto Rico

Se ha documentado que aproximadamente el $97 \%$ de los puertorriqueños se autodenominan cristianos (Pew Research Center's Forum on Religion \& Public Life, 2012) y que alrededor del $93 \%$ de los puertorriqueños considera a la religión como un aspecto importante en su vida (The Association of Religion Data Archives, 2003). Por su parte, González-Rivera, Veray-Alicea y Rosario-Rodríguez (2016) encontraron que el 
96\% de los profesionales de ayuda de Puerto Rico (psicólogos, consejeros y profesionales del trabajo social) muestran una actitud favorable hacia integrar la espiritualidad en la psicoterapia y la consejería. Por consiguiente, consideramos relevante para el avance de la psicología clínica en Puerto Rico la integración de recursos basados en la evidencia asociados con el tema de la religión y la espiritualidad con el objetivo de evaluar su impacto en el bienestar psicológico y físico de los pacientes interesados en este tipo de tópico y ofrecer servicios a poblaciones con necesidades espirituales y religiosas (Pagán-Torres et al., 2017).

En Puerto Rico, Reyes-Estrada, RiveraSegarra, Ramos-Pibernus, Rosario-Hernández y Rivera-Medina (2014) desarrollaron y validaron la primera escala de religiosidad en la población puertorriqueña con 140 participantes. La escala obtuvo una consistencia interna alfa de Cronbach de .97 para la escala total, mientras que la consistencia interna de las subescalas fluctuó entre .94 y .97. Por otra parte, González-Rivera (2017) desarrolló una escala de religiosidad personal para medir estrictamente el constructo religiosidad sin contemplar elementos asociados con la espiritualidad. Dicha medida está compuesta por 21 reactivos divididos en dos factores: creencias/ actitudes y prácticas religiosas. Este instrumento obtuvo un índice de consistencia interna alfa de Cronbach de .96 .

El único instrumento de afrontamiento religioso traducido al español y validado en Puerto Rico es el Brief Religious of Coping Scale (BRCOPE; Colón-Rivera, 2014). Sus propiedades psicométricas se evaluaron en una muestra de 226 adultos puertorriqueños. La escala consta de 14 reactivos en formato Likert con cuatro puntos que fluctúan desde Para nada (1) a Muchísimo (5). Los reactivos se encuentran distribuidos en dos dimensiones clasificadas como afrontamiento religioso positivo (ARP) y afrontamiento religioso negativo (ARN). Las propiedades psicométricas del instrumento revelaron un coeficiente de confiabilidad alfa de Cronbach para la subescala de ARP de .93, mientras que para la subescala de ARN fue de .88. Esta medida tiene la característica particular de medir estrategias de afrontamiento religioso tanto positivas como negativas.

Sin embargo, a pesar de que existe una escala de afrontamiento religioso traducida $y$ validada en el contexto puertorriqueño, dicha medida no se enfoca en medir estrategias internas (cognitivas) ni externas (conductuales) de afrontamiento religioso, sino que examina estilos de afrontamiento religioso (estilo positivo y estilo negativo). La razón de ello se debe a que dicho instrumento está fundamentado en la visión teórica de Pargament (1997). Ciertamente, las estrategias internas y externas deben considerarse como un método de prevención y utilidad para afrontar eventos estresantes en la vida. Por tal razón, entendemos que nuestro marco teórico, orientado a estrategias internas y externas, guarda de forma simultánea similitudes y diferencias con el BRCOPE (Colón-Rivera, 2014). Esto permitirá que futuras investigaciones puedan tener una mayor comprensión del afrontamiento religioso en Puerto Rico y examinar el mismo desde sus distintas dimensiones.

\section{Propósito del estudio}

El propósito principal de esta investigación fue desarrollar, validar y examinar las propiedades psicométricas del Inventario de Estrategias de Afrontamiento Religioso (IEAR) utilizando estadísticas avanzadas. Específicamente, esta investigación cuenta con cuatro objetivos principales:

1. Analizar la estructura factorial del 
Inventario de Estrategias de Afrontamiento Religioso (IEAR) mediante análisis factorial confirmatorio con ecuaciones estructurales.

2. Analizar la capacidad de discriminación de los ítems del IEAR.

3. Analizar la confiabilidad del IEAR y sus factores mediante el índice de consistencia interna alfa de Cronbach y la confiabilidad compuesta.

4. Analizar la validez convergente $y$ divergente de los factores del IEAR mediante el análisis de varianza media extraída.

\section{Método}

Diseño

Esta investigación tiene un diseño de estudio instrumental (Montero \& León, 2007) en el que se analizaron las propiedades psicométricas del IEAR mediante análisis factorial confirmatorio. De esta forma se puso a prueba la estructura factorial del instrumento y se cumplieron los objetivos propuestos.

\section{Participantes}

Se trabajó con una muestra no probabilística constituida por 350 participantes, con una edad promedio de 43.25 años $(\mathrm{DE}=14.126)$. Como criterio para seleccionar el tamaño de la muestra, seguimos las recomendaciones de MacCallum, Widaman, Preacher y Hong (2001), quienes sugieren un radio conservador de 20 participantes por cada ítem de la escala por evaluar. En nuestro caso, la escala por examinar comprende 15 ítems, lo que sugiere un mínimo de 300 participantes. En la Tabla 1 se presentan los datos sociodemográficos de los participantes. Para participar en este estudio, se establecieron los siguientes criterios de inclusión: (1) ser mayor de
21 años de edad, (2) tener la capacidad de leer en español, y (3) ser residente en Puerto Rico.

Tabla 1 Información sociodemográfica de la muestra.

\begin{tabular}{|c|c|c|}
\hline Variables & $f$ & $\%$ \\
\hline \multicolumn{3}{|l|}{ Sexo } \\
\hline Femenino & 211 & 60.3 \\
\hline Masculino & 139 & 39.7 \\
\hline \multicolumn{3}{|l|}{ Preparación Académica } \\
\hline Escuela superior o menos & 26 & 7.4 \\
\hline Grado asociado / técnico & 50 & 14.3 \\
\hline Bachillerato & 162 & 46.3 \\
\hline Maestría & 89 & 25.4 \\
\hline Doctorado o grado equivalente & 23 & 6.8 \\
\hline \multicolumn{3}{|l|}{ Estado civil } \\
\hline Soltero/a & 136 & 38.9 \\
\hline Casado/a & 133 & 38.0 \\
\hline Divorciado/a & 48 & 13.7 \\
\hline Viudo/a & 8 & 2.3 \\
\hline Convivencia & 25 & 7.1 \\
\hline \multicolumn{3}{|l|}{ Religión } \\
\hline Católico/a & 111 & 31.7 \\
\hline Cristiano Protestante & 162 & 46.3 \\
\hline Budista & 4 & 1.1 \\
\hline Ateo/agnóstico & 15 & 4.3 \\
\hline Ninguna & 31 & 8.9 \\
\hline Otra & 27 & 7.7 \\
\hline
\end{tabular}

Nota. $N=350$.

\section{Instrumentos}

Cuestionario de Datos Generales. Para identificar las características sociodemográficas de la muestra desarrollamos un cuestionario de datos generales a través del cual se recogió información importante relacionada con la edad, sexo, religión y preparación académica.

Inventario de Estrategias de Afrontamiento Religioso (IEAR). Este instrumento fue desarrollado por los investigadores para medir estrategias internas (cognitivas) y externas 
(conductuales) de afrontamiento religioso. La versión original del instrumento estaba constituida por 15 estrategias de afrontamiento religioso -fundamentadas en la literatura científica-, comúnmente utilizadas en el manejo de eventos negativos y situaciones estresantes (p. ej., Continuar confiando en Dios; Encontrar fortaleza en la oración; Buscar apoyo en mi comunidad de fe). El instrumento tiene una escala de respuesta tipo Likert de cinco puntos: 0 (En absoluto), 1 (Un poco), 2 (Bastante), 3 (Mucho) y 4 (Totalmente). El puntaje más bajo que se puede obtener en la versión original es 0 y el más alto es 60, tal que una mayor puntuación indica un mayor uso de estrategias de afrontamiento religioso internas o externas.

\section{Procedimientos Generales}

La fase de reclutamiento se llevó a cabo de forma electrónica y presencial durante el mes de noviembre de 2017. Para proteger y garantizar los derechos de los participantes utilizamos una hoja de consentimiento informado donde se notificó lo siguiente: (a) propósito del estudio, (b) naturaleza voluntaria del estudio, (c) posibles riesgos y beneficios, (d) su derecho a retirarse del estudio en cualquier momento, (e) institución de procedencia y (f) datos de identificación y contacto de los investigadores.

Se reclutaron personas de forma grupal e individual en dos centros universitarios de la zona metropolitana de Puerto Rico. En todos los casos se ofreció a los participantes información básica del propósito de la investigación y se informó sobre los criterios de inclusión. Una vez que leyeron el consentimiento informado $y$ lo firmaron aceptando participar voluntariamente del estudio se les facilitó los instrumentos. Luego de completar los cuestionarios, entregaron los mismos en un sobre sellado que no podía ser pareado con el consentimiento informado. El $27 \%(\mathrm{n}=95)$ de la muestra fue reclutado de forma presencial.

El reclutamiento electrónico comenzó con un anuncio pagado en Facebook. Le solicitamos a los administradores de esta red social que promocionaran el anuncio entre adultos mayores de 21 años de edad residentes de Puerto Rico. El anuncio alcanzó 40,564 personas y fue compartido por 110 personas en Facebook, provocando un efecto "bola de nieve" en las redes sociales. Este anuncio redirigía a las participantes a una encuesta en línea disponible en la plataforma PsychData, la cual estuvo activa durante un mes (noviembre 2017). Esta plataforma generó una base de datos que fue integrada en una sola base con los cuestionarios recopilados de forma presencial. El $73 \%(n=255)$ de la muestra se obtuvo mediante este proceso.

\section{Estrategia de Análisis de Datos}

Una vez recopilados los datos, examinamos las propiedades psicométricas del IEAR partiendo de las percepciones de los participantes sobre cada uno de los ítems del instrumento. Para esto, utilizamos el sistema estadístico IBM SPSS v.24 (IBM Corp., 2016). En este programa realizamos análisis descriptivos (medias y desviaciones típicas), análisis de distribución de datos (curtosis, asimetría, Kolmogorov-Smirnov, Shapiro-Wilk), análisis de reactivos para conocer su índice de discriminación y análisis de confiabilidad.

Para el análisis factorial confirmatorio utilizamos AMOS Graphic v.24 (Arbuckle, 2016), con el método de estimación de máxima verosimilitud y el programa estadístico STATA 15 (StataCorp., 2017) para evaluar el ajuste de los modelos de ecuaciones estructurales con las correcciones de Satorra y Bentler (2001). 
Primero, analizamos dos modelos de ecuaciones estructurales: (1) un modelo base conformado por los 15 ítems originales del IEAR (MB) y (2) un segundo modelo en el que los 15 ítems convergían en dos factores, estrategias internas y estrategias externas (M1). Sin embargo, para evitar la redundancia entre los parámetros de estimación y obtener un modelo más frugal, examinamos los coeficientes de regresión de cada reactivo y revisamos la covarianza residual estandarizada en las matrices dentro de los estimados, como recomienda Byrne (2010). Esto nos permitió identificar los valores problemáticos y eliminar los ítems que generaban dificultades en el ajuste del modelo. Este procedimiento redujo la escala a 12 ítems, lo que permitió evaluar un tercer modelo de dos factores con seis ítems cada uno (M2).

Para examinar los resultados del análisis factorial confirmatorio, utilizamos los siguientes índices de ajuste absoluto (Byrne, 2010): ChiCuadrado $\left(\chi^{2}\right)$ y Error Medio Cuadrático de Aproximación (RMSEA). En cuanto al RMSEA, valores menores a .08 indican un ajuste aceptable del modelo, mientras que valores menores a .05 indican un buen ajuste del modelo. Debido a que el Chi-Cuadrado es sensible al tamaño de la muestra, utilizamos otros índices de ajuste al momento de evaluar el modelo: el Índice Tucker-Lewis (TLI), el Índice de Ajuste Comparativo (CFI) y el Criterio de Información de Akaike (AIC). Siguiendo las indicaciones de Byrne (2010), se consideró que valores iguales o mayores a .90 en los índices TLI y CFI reflejan un ajuste aceptable, mientras que valores iguales o mayores a .95 muestran un buen ajuste del modelo. Por su parte, el AIC es utilizado para examinar la complejidad/ parsimonia de los modelos y comparar los mismos, por lo tanto, se prefiere el modelo con el menor índice (Schumacker \& Lomax, 2010).

Una vez obtenido el modelo con los mejores índices de ajuste (M2), se procedió a hacer un análisis de discriminación de los ítems a través de la correlación ítem-total. Se consideró que aquellos reactivos con intercorrelaciones mayores a .30 poseen índices aceptables de discriminación (Kline, 2005). A su vez, calculamos la confiabilidad del IEAR utilizando el coeficiente alfa de Cronbach y la confiabilidad compuesta; ambos índices debían ser mayores a 70 (Kline, 2000; Raykov \& Shrout, 2002). Además, siguiendo las recomendaciones de Fornell y Larcker (1981), examinamos la validez convergente y discriminante del IEAR mediante la Varianza Media Extraída (VME); la cual estima el promedio de varianza media explicada por los ítems que componen la escala. Para apoyar la validez convergente, la VME debe ser igual o mayor a .50. Por último, Fornell y Larcker (1981) explican que, para determinar la validez discriminante de cada dimensión, las correlaciones cuadradas de los factores del instrumento deben ser menores que el VME.

\section{Resultados \\ Análisis Descriptivos y de Distribución}

En primer lugar, obtuvimos las medias y desviaciones estándar para cada ítem del IEAR para analizar las propiedades de distribución de la escala. Las medias de los ítems fluctuaron entre 1.67 y 3.30 , con desviaciones estándar que fluctuaron entre 1.291 y 1.671. Como muestra la Tabla 2, las pruebas de normalidad KolmogorovSmirnov y Shapiro-Wilk evidencian que los datos no se distribuyen de forma normal $(p<.001)$. Debido a la falta de normalidad en los datos utilizamos las correcciones de Satorra y Bentler (2001) para calcular el ajuste de los modelos de ecuaciones estructurales, ya que la no-normalidad de los datos altera los errores de estimación y el ajuste global del modelo. 
Tabla 2 Estadísticos de descriptivos y de distribución para los ítems de la versión final.

\begin{tabular}{ccccccc}
\hline Ítem & Media & $\begin{array}{c}\text { Desviación } \\
\text { Estándar }\end{array}$ & Asimetría & Curtosis & $\begin{array}{c}\text { Kolmogorov- } \\
\text { Smirnov }\end{array}$ & Shapiro-Wilk \\
\hline REL1 & 3.30 & 1.29 & -1.66 & 1.24 & .43 & .59 \\
REL2 & 3.10 & 1.40 & -1.29 & .10 & .38 & .66 \\
REL3 & 3.04 & 1.39 & -1.15 & -.19 & .36 & .69 \\
REL4 & 3.11 & 1.39 & -1.31 & .21 & .38 & .66 \\
REL5 & 2.89 & 1.37 & -.93 & -.54 & .30 & .76 \\
REL6 & 3.12 & 1.36 & -1.36 & .39 & .36 & .67 \\
REL8 & 2.09 & 1.64 & -.35 & -1.66 & .22 & .81 \\
REL10 & 2.14 & 1.67 & -.08 & -1.68 & .24 & .80 \\
REL12 & 2.41 & 1.59 & -.39 & -1.45 & .24 & .81 \\
REL13 & 2.34 & 1.51 & -.28 & -1.43 & .21 & .84 \\
REL14 & 2.21 & 1.61 & -.15 & -1.61 & .23 & .82 \\
REL15 & 1.67 & 1.60 & .38 & -1.49 & .23 & .81 \\
\hline
\end{tabular}

Nota. Error estándar de la asimetría $=.13$; Error estándar de la curtosis $=.26$. Grados de libertad Kolmogorov-Smirnov y Shapiro-Wilk $=350$, todos los valores $\mathrm{p}<.001$.

\section{Análisis factorial confirmatorio}

Luego, evaluamos la estructura factorial del IEAR mediante análisis factorial confirmatorio con ecuaciones estructurales utilizando el método de estimación de máxima verosimilitud. En este proceso examinamos tres modelos comparativos. El primer modelo que sometimos a prueba fue el modelo base (MB) en el que los 15 ítems del IEAR convergían en un solo factor latente. Este modelo no presentó un buen ajuste a los datos, $\chi^{2}=1262.53(90) p<.001$, RMSEA $=.19$, CFI $=.81, \mathrm{TLI}=.78, \mathrm{AIC}=1322.53, \chi^{2}$ corregido $=$ 850.03 (90) $p<.001$, RMSEA corregido $=.16$, CFI corregido $=.83$, TLI corregido $=.83$. Esto sugiere que la estructura factorial de la escala no está conformada por un solo factor. Ante esto, examinamos un segundo modelo de dos factores (M1), estrategias internas (ocho ítems) y externas (siete ítems) de afrontamiento religioso. Este modelo tampoco evidenció un ajuste adecuado de los datos, $\chi^{2}=815.16(89) p<.001$, RMSEA $=.15$, $\mathrm{CFI}=.88, \mathrm{TLI}=.86, \mathrm{AIC}=877.16, \chi^{2}$ corregido
$=554.87(89) p<.001$, RMSEA corregido $=.12$, CFI corregido $=.90$, TLI corregido $=.88$.

Con la intención de lograr mayor parsimonia en el modelo y mejores índices de ajuste revisamos la covarianza residual estandarizada en las matrices dentro de los estimados (Byrne, 2010), identificamos los valores problemáticos y eliminamos los ítems que dificultaban el ajuste del modelo. Luego de eliminar los ítems 7, 9 y 11, obtuvimos el Modelo 2 hipotetizado (M2), conformado también por dos factores latentes con seis ítems cada uno (ver Figura 1), el cual presentó un buen ajuste en todos los índices, $\chi^{2}=$ $162.670(46) p<.001, \mathrm{RMSEA}=.08, \mathrm{CFI}=.97$, $\mathrm{TLI}=.96, \mathrm{AIC}=226.67, \chi^{2}$ corregido $=122.14$ (46) $p<.001$, RMSEA corregido $=.07$, CFI corregido $=.98$, TLI corregido $=.97$. Siguiendo las recomendaciones propuestas por Schumacker y Lomax (2010), utilizamos el AIC para la comparación de los tres modelos hipotetizados, ya que los valores de $\chi^{2}$ fueron estadísticamente significativos. El M2 presentó un índice menor $(\mathrm{AIC}=226.67)$ que los otros dos modelos (ver Tabla 3). 
Tabla 3

Bondades de ajuste para los modelos de análisis de factores confirmatorios.

\begin{tabular}{lllllllllll}
\hline Modelo & $\chi^{2}$ & $\chi_{\text {corr }}^{2}$ & gl & RMSEA & RMSEA $_{\text {corr }}$ & CFI & CFI $_{\text {corr }}$ & TLI & TLI $_{\text {corr }}$ & AIC \\
\hline MB & 1262.53 & 850.03 & 90 & .19 & .16 & .81 & .83 & .78 & .83 & 1322.53 \\
M1 & 815.16 & 554.87 & 89 & .15 & .12 & .88 & .90 & .86 & .88 & 877.16 \\
M2 & 162.670 & 122.14 & 46 & .08 & .07 & .97 & .98 & .96 & .97 & 226.67 \\
\hline
\end{tabular}

Nota. $\chi^{2}=$ chi cuadrado; $\chi 2$ corr $=$ chi cuadrado corregido; $\mathrm{gl}=$ grados de libertad; RMSEA $=$ Error Medio Cuadrático de Aproximación; RMSEAcorr $=$ Error Medio Cuadrático de Aproximación corregido; CFI = Índice de Ajuste Comparativo; CFIcorr = Índice de Ajuste Comparativo corregido; TLI = Índice Tucker-Lewis; TLIcorr $=$ Índice Tucker-Lewis corregido; AIC = Akaike Information Criterion; Todos los estadísticos $\chi^{2}$ y $\chi^{2} \operatorname{corr}$ son significativos, $\mathrm{p}<.001$.

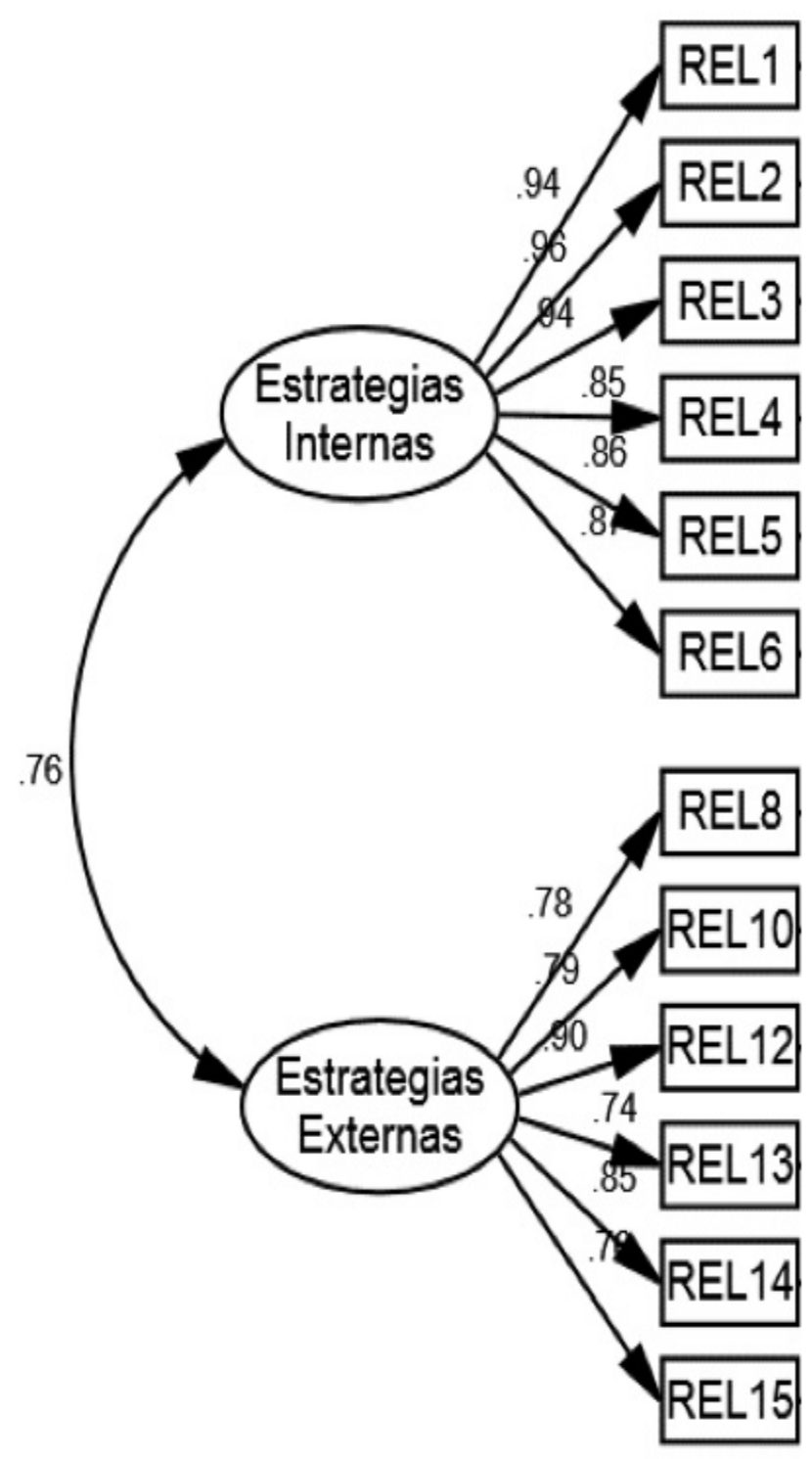

Figura 1

Modelo final del IEAR (M2).
Análisis de los Ítems

Con los 12 ítems que conformaron el M2, realizamos dos análisis de discriminación a través del índice de correlación ítem-total $\left(r_{\mathrm{bis}}\right)$ : uno para calcular el índice del ítem al compararlo con la escala total y otro para calcular el índice de discriminación delítem en su respectiva subescala. Además, calculamos varianza explicada de los factores/subescalas y escala total en los ítems. La Tabla 4 presenta los índices de discriminación de la versión final del IEAR, los cuales están por encima del mínimo recomendado de .30 (Kline, 2005).

\section{Análisis de Confiabilidad}

Posteriormente, analizamos la confiabilidad compuesta para la versión final de dos factores. Para medir la confiabilidad de los factores y la escala total utilizamos dos métodos: el coeficiente Alfa de Cronbach y la división en mitades de Spearman-Brown. Estos tres índices superaron los valores mínimos recomendados por la literatura. La Tabla 5 resume los valores de confiabilidad compuesta para cada dimensión, así como del instrumento en su totalidad. 
Tabla 4

Índices de discriminación, varianza explicada y coeficientes de regresión de los ítems.

\begin{tabular}{|c|c|c|c|c|c|}
\hline \multirow{2}{*}{ Ítems } & \multicolumn{2}{|c|}{ Escala Total } & \multicolumn{2}{|c|}{ Factores } & \multirow[b]{2}{*}{$\boldsymbol{R}^{2}$} \\
\hline & $r_{b i s}$ & $\boldsymbol{R}^{2}$ & $r_{b i s}$ & $\boldsymbol{\beta}$ & \\
\hline 1. Continuar confiando en Dios. & .79 & .86 & .90 & .94 & .95 \\
\hline 2. Buscar consuelo en Dios. & .86 & .90 & .93 & .96 & .95 \\
\hline 3. Encontrar fortaleza en la oración. & .85 & .85 & .90 & .94 & .95 \\
\hline 4. Pensar que Dios siempre tiene el control. & .78 & .78 & .86 & .85 & .96 \\
\hline 5. Aferrarme a mis creencias religiosas y espirituales. & .82 & .74 & .83 & .86 & .96 \\
\hline 6. Creer que Dios tiene un propósito con esta situación. & .79 & .79 & .88 & .87 & .96 \\
\hline 7. Buscar apoyo en mi comunidad de fe. & .79 & .73 & .81 & .78 & .70 \\
\hline 8. Asistir a la iglesia o al templo. & .76 & .71 & .79 & .79 & .70 \\
\hline 9. Leer textos sagrados de mi religión (ej. Biblia). & .82 & .74 & .82 & .90 & .70 \\
\hline 10. Leer otros libros de crecimiento espiritual. & .64 & .53 & .67 & .73 & .52 \\
\hline 11. Escuchar música sacra o religiosa. & .76 & .68 & .80 & .85 & .66 \\
\hline 12. Recibir consejería o dirección espiritual. & .71 & .68 & .80 & .76 & .66 \\
\hline
\end{tabular}

Nota. rbis = Índice de discriminación; R2 = Varianza explicada; $\beta$ = coeficientes de regresión; Ítems del 1 al 6 = Factor estrategias internas; Ítems del 7 al $12=$ Factor estrategias externas.

Evidencias de la validez convergente y discriminante

Por último, analizamos la validez convergente y discriminante mediante la Varianza Media Extraída (VME), la cual indica la proporción de la varianza en los ítems explicada por el factor latente. Para considerar que existe validez convergente, la VME debe superar el valor de .50 (Bagozzi \& Yi, 1988). En cuanto a la vali- dez discriminante, los dos factores no comparten una cantidad sustancial de varianza entre sí. La varianza compartida entre los factores es de .58. Según Fornell y Larcker (1981), la varianza compartida entre dos factores siempre es menor a la varianza explicada por cada uno de los factores (VME). Según los valores que se presentan en la Tabla 4, el IEAR cumple con los criterios de validez convergente y validez discriminante. Además, analizamos la relación entre los factores del IEAR mediante la correlación $r$ de Pearson (véase Tabla 6).

Tabla 5

Medias, desviaciones estándar, coeficientes alfa y fiabilidad compuesta.

\begin{tabular}{llllll}
\hline Factor & M & DE & $\boldsymbol{\alpha}$ & Spearman-Brown & FC \\
\hline Estrategias Internas & 18.57 & 7.55 & .96 & .95 & .94 \\
Estrategias Externas & 12.85 & 8.21 & .92 & .91 & .81 \\
Escala Total & 31.42 & 14.62 & .95 & .84 & \\
\hline
\end{tabular}

Nota. $\mathrm{M}=$ media; $\mathrm{DE}=$ desviación estándar; $\alpha=$ alfa de Cronbach; $\mathrm{FC}=$ fiabilidad compuesta $(\mathrm{n}=350)$. 
Tabla 6

Evidencia de validez, varianza media extraída y correlaciones.

\begin{tabular}{lllll}
\hline Factor & Ítems & $\boldsymbol{V M E}$ & $\mathbf{1}$ & $\mathbf{2}$ \\
\hline $\begin{array}{l}\text { Estrategias Internas } \\
\text { de Afrontamiento }\end{array}$ & 6 & .82 & 1 & $.76^{*}$ \\
$\begin{array}{l}\text { Estrategias Externas } \\
\text { de Afrontamiento }\end{array}$ & 6 & .65 & $.72 *$ & 1 \\
\hline
\end{tabular}

Nota. Ítems = cantidad de ítems del factor; VME = varianza media extraída; $*=p<.001$. El valor sobre la diagonal representa la correlación entre los factores latentes, mientras que el valor por debajo de la diagonal representa la correlación de las puntuaciones directas.

\section{Discusión}

El presente estudio tuvo como propósito desarrollar, validar y examinar las propiedades psicométricas del Inventario de Estrategias de Afrontamiento Religioso (IEAR) utilizando estadísticas avanzadas en una muestra de adultos en Puerto Rico.A partir de los resultados obtenidos, se puede establecer que el IEAR posee adecuadas características psicométricas para medir estrategias internas y externas de afrontamiento religioso en adultos puertorriqueños. Estos dos factores miden de forma confiable un elemento particular del constructo, sin que haya redundancia con el otro factor. Además, los índices de confiabilidad obtenidos sugieren, según lo establece Kline (2000), que el IEAR cuenta con la suficiente consistencia interna para ser utilizado como instrumento de medición científica en investigaciones en Puerto Rico y América Latina, o como método de cernimiento en contextos clínicos puertorriqueños.

De forma general, el análisis factorial confirmatorio mostró que el modelo hipotetizado presenta un ajuste satisfactorio con los datos y confirmó la estructura bifactorial del IEAR. Estos resultados son congruentes con los obtenidos en otros estudios donde se han desarrollado instrumentos para medir afrontamiento religioso o religiosidad desde una perspectiva bifactorial (Colón-Rivera, 2014; González-Rivera, 2017; Pargament etal., 2011). En cuanto a la confiabilidad del IEAR, se obtuvieron índices superiores a los evidenciados por escalas bifactoriales similares en Puerto Rico (Colón-Rivera, 2014; GonzálezRivera, 2017) y Argentina (Mezzadra \& Simkin, 2017). Asimismo, las correlaciones de cada ítem con la puntuación total manifiestan una consistencia interna notable.

En términos de las implicaciones teóricas del estudio, los hallazgos psicométricos demostraron que la versión final del IEAR replica las dos dimensiones consideradas por los autores en la construcción teórica del instrumento y en la redacción de los ítems: estrategias internas (cognitivas) y estrategias externas (conductuales) de afrontamiento religioso. Esta concepción teórica del afrontamiento religioso guarda estrecha relación con los fundamentos $\mathrm{y}$ supuestos teóricos del modelo de afrontamiento de Lazarus y Folkman (1986). Estos autores, al igual que nosotros, conciben el afrontamiento y el manejo de situaciones adversas desde una perspectiva cognitivo-conductual. Es decir, las destrezas de afrontamiento religioso suponen esfuerzos cognitivos para manejar pensamientos maladaptativos y eventos privados desagradables, así como esfuerzos conductuales que faciliten la búsqueda de recursos y herramientas externas que empoderen y motiven a la persona en su proceso de recuperación y ajuste.

En el caso de Puerto Rico, como en América Latina, donde un número considerable de personas se identifican como practicantes y/o creyentes de algún credo religioso, es común observar cómo estos integran estrategias religioso-espirituales en sus esfuerzos de ajuste y adaptación (González- 
Rivera, 2015). De hecho, Quiceno y Vinaccia (2011) explican que la literatura científica es concluyente al plantear que, ante circunstancias difíciles de la vida, el afrontamiento religioso sirve para atenuar la presencia de emociones negativas, manejar el estrés y mejorar la calidad de vida. En este contexto, nuestra investigación aporta y propone a la comunidad científica un instrumento válido y confiable que examina desde sus dos principales dimensiones, las estrategias de afrontamiento religioso de los individuos. Estos hallazgos robustecen la teoría bifactorial de afrontamiento religioso que sustenta los supuestos teóricos del instrumento.

En cuanto a las implicaciones prácticas del estudio, demostramos que la versión final del IEAR puede ser utilizada para el desarrollo de nuevas investigaciones en el campo de la psicología de la religión y la espiritualidad en el Caribe y América Latina. Además, facilitará a los psicoterapeutas la realización de cernimientos y avalúos para entender la manera en que la espiritualidad y las destrezas de afrontamiento religioso pueden beneficiar los procesos terapéuticos de los pacientes. El lenguaje sencillo y la estructura breve del instrumento hacen del IEAR una escala de fácil administración, evaluación e interpretación. En este sentido, este instrumento se constituye como una herramienta práctica y efectiva en el quehacer clínico e investigativo de los psicólogos y demás profesionales de la conducta.

Sobre el uso de la escala, debemos señalar que los 12 ítems de la versión final del IEAR se distribuyen en dos factores (seis ítems por cada uno) fundamentados en el marco teórico bifactorial de afrontamiento religioso desarrollado por los autores (estrategias internas y externas). Las puntuaciones del IEAR pueden ser calculadas de dos formas: mediante la sumatoria de los 12 ítems del instrumento final para obtener un índice general de afrontamiento religioso (útil para estudiar correlaciones entre esta variable y otros constructos psicológicos) y mediante la sumatoria de los seis ítems de cada factor por separado para obtener una puntuación específica por tipo de estrategia, interna o externa. El orden de los ítems en la versión final se determinó por categoría, los primeros seis ítems corresponden a las estrategias internas, y los últimos seis, a las estrategias externas. Dados los coeficientes correlacionales moderados y estadísticamente significativos entre las subescalas, la suma de las puntuaciones en cada una de ellas se considera un índice general de afrontamiento religioso. Las puntuaciones posibles fluctúan de 0 a 48 . Se entiende que a mayor puntuación obtenida, mayor cantidad de estrategias de afrontamiento religioso utiliza la persona.

\section{Limitaciones y fortalezas}

Al igual que toda investigación, nuestro estudio no está exento de limitaciones. Primero, la muestra fue no probabilística $y$ no fue aleatoria; sin embargo, fue amplia y heterogénea. Segundo, no se pudo establecer la confiabilidad del instrumento a través del tiempo, sólo se pudo evaluar a través de sus elementos. No obstante, las técnicas avanzadas utilizadas en el estudio brindan robustez empírica a nuestros resultados. Por último, el procedimiento para recoger los datos no fue estandarizado, esto puede afectar las medias del estudio y aumentar el error típico de medición.

A pesar de sus limitaciones, la presente investigación tiene varias fortalezas que vale la pena enumerar. En primer lugar, es el primer instrumento desarrollado y validado en Puerto Rico para medir el afrontamiento religioso desde una perspectiva cognitivo-conductual y para el que se toman en consideración las particularidades de 
la cultura caribeña y latinoamericana. Segundo, robustece la teoría bifactorial de afrontamiento religioso, al proveer evidencia teórica y empírica sobre la estructura factorial del instrumento. Por último, ofrece a la comunidad científica hispanoparlante un instrumento confiable y válido que enriquecerá las investigaciones dirigidas a entender con mayor profundidad la relación entre el afrontamiento religioso-espiritual y la salud mental.

\section{Futuras investigaciones}

En cuanto a recomendaciones para futuras investigaciones, aconsejamos administrar el IEAR a otra muestra de participantes para realizar el proceso de validación cruzada. También se debe examinar la confiabilidad temporal a través de la técnica de test-retest. Además, recomendamos que el IEAR sea validado en otras poblaciones latinoamericanas para evaluar sus propiedades psicométricas en distintos contextos nacionales e internacionales. Por último, recomendamos la utilización del IEAR para el desarrollo de nuevas investigaciones sobre el afrontamiento religiosoespiritual en Puerto Rico y América Latina.

\section{Conclusión General}

La construcción y validación del Inventario de Estrategias de Afrontamiento Religioso (IEAR) hace dos importantes aportaciones al quehacer psicológico en Puerto Rico y al desarrollo de la psicología de la religión y la espiritualidad en América Latina. En primer lugar, el IEAR tiene la capacidad de contribuir al aumento de investigaciones científicas en el contexto sociocultural puertorriqueño y latinoamericano que permitan entender con mayor profundidad el efecto del afrontamiento religioso, tanto interno como externo, en la salud mental de la población. Por otra parte, en el contexto clínico el IEAR contribuye como una herramienta práctica para quelos psicólogos puedan incorporary tratar temas asociados con la religiosidad y la espiritualidad en el proceso terapéutico de manera sistemática. Estas dos aportaciones incrementan el valor y la necesidad de este instrumento en la práctica de la psicología, tanto clínica como investigativa.

\section{Referencias}

Ano, G. G., \& Vasconcelles, E. B. (2005). Religious coping and psychological adjustment to stress: A metaanalysis. Journal of Clinical Psychology, 61(4), 461480. doi: 10.1002/jclp.20049

Arbuckle, J. L. (2016). Amos (Versión 24.0) [software de cómputo]. Chicago, IL: IBM SPSS.

Asociación Americana de Psicología (2010). Diccionario conciso de Psicología. México: Manual Moderno.

Asociación Americana de Psiquiatría (2013). Manual diagnóstico y estadístico de los trastornos mentales: DSM-5. Washington, DC: Autor.

Bagozzi, R. P., \& Yi, Y. (1988). On the evaluation of structural equation models. Journal of the Academy of Marketing Science, 16(1), 74-94. doi: 10.1007/ BF02723327

Beck, J. (2011). Cognitive-Behavior Therapy. Basics and beyond. New York, NY: Guilford.

Bonelli, R. M., \& Koenig, H. G. (2013). Mental disorders, religion and spirituality 1990 to 2010: A systematic evidence-based review. Journal of Religion and Health, 52(2), 657-673. doi: 10.1007/s10943-0139691-4

Byrne, B. M. (2010). Structural equation modeling with AMOS: Basic concepts, applications, and programming. New York: Psychology Press.

Carver, C. S., Scheier, M. F., \& Weintraub, J. K. (1989). Assessing coping strategies: A theoretically based 
approach. Journal of Personality and Social Psychology, 56(2), 267-283. doi: 10.1037/00223514.56.2.267

Colón-Rivera, Y. (2014). Traducción, adaptación cultural $y$ validación del Brief Religious Coping Scale en una muestra de puertorriqueños/as. (Disertación doctoral). Ponce Health Sciences University, Ponce, Puerto Rico.

Endler, N. S., \& Parker, J. D. (1999). Coping Inventory for Stressful Situations (CISS): Manual. Toronto, ON, Canadá: Multi-Health Systems.

Folkman, S., \& Lazarus, R. S. (1980). An analysis of coping in a middle-aged community sample. Journal of Health and Social Behavior, 21(3), 219-239. doi: $10.2307 / 2136617$

Folkman, S., \& Lazarus, R. S. (1988). Manual for the Ways of Coping Questionnaire. Palo Alto, California: Consulting Psychologists Press.

Fornell, C., \& Larcker, D. F. (1981). Evaluating structural equation models with unobservable variables and measurement error. Journal of Marketing Research, 18(1), 39-50. Recuperado de http://www.jstor.org/ stable/3151312

González-Rivera, J. A. (2015). Espiritualidad en la clínica: Integrando la espiritualidad en la psicoterapia y la consejería. San Juan, PR: Ediciones Psicoespiritualidad.

González-Rivera, J. A. (2017). Propiedades psicométricas de la Escala de Religiosidad Personal en una muestra de adultos en Puerto Rico. Revista Electrónica de Psicología Iztacala, 20(4), 1386-1406. Recuperado de http://www.iztacala.unam.mx/carreras/psicologia/ psiclin/principal.html

González-Rivera, J. A., Veray-Alicea, J., \& RosarioRodríguez, A. (2016). Actitudes hacia la integración de la espiritualidad en las profesiones de ayuda: Estudio exploratorio. Revista Griot, 9(1), 57-67. Recuperado de http://revistas.upr.edu/index.php/grio t/

Green, M., \& Elliott, M. (2010). Religion, health and psychological well-being. Journal of Religion and
Health, 49(2), 149-163. doi: 10.1007/s10943-0099242-1

IBM Corp (2016). IBM SPSS Statistics for Windows, Version 24.0. [software de cómputo]. Armonk, NY: IBM Corp.

Kline, P. (2000). The Handbook of Psychometric Testing. New York: Routledge.

Kline, T. J. (2005). Psychological testing: A practical approach to design and evaluation. Thousand Oaks: Sage. doi: 10.4135/9781483385693

Koenig, H. (2008). Medicine, religion and health: Where science and spirituality meet. West Conshohocken, PA: Temple Foundation Press. doi: 10.1097/01. nmd.0000349943.48219.f5

Koenig, H. G. (2012). Religion, spirituality, and health: The research and clinical implications. International Scholarly Research Network, 2012, 1-33. doi: 10.5402/2012/278730

Koenig, H. G. (2015). Religion, spirituality, and health: A review and update. Advances in Mind-Body Medicine, 29(3), 19-26. Recuperado de https:// www.readbyqxmd.com/read/26026153/religionspirituality-and-health-a-review-and-update

Lazarus, R. S., \& Folkman, S. (1986). Estrés y procesos cognitivos. Barcelona: Martínez Roca.

MacCallum, R. C., Widaman, K. F., Preacher, K. J., \& Hong, S. (2001). Sample size in factor analysis: The role of model error. Multivariate Behavioral Research, 36(4), 611-637. doi: 10.1207/S15327906MBR3604_06

Mezzadra, J., \& Simkin, H. (2017). Validación de la escala abreviada de afrontamiento religioso Brief-RCOPE en el contexto argentino en estudiantes de confesión católica. Revista Evaluar, 17(1), 18-28. Recuperado de https://revistas.unc.edu.ar/index.php/revaluar

Montero, I., \& León, O. G. (2007). A guide for naming research studies in psychology. International Journal of Clinical and Health Psychology, 7(3), 847-862. Recuperado de http://www.aepc.es/ijchp/GNEIP07_ es.pdf

Pagán-Torres, O. M., Reyes-Estrada, M., \& CumbaAvilés, E. (2017). Religión, espiritualidad y terapia 
cognitivo conductual: Una reseña actualizada. Salud y Conducta Humana, 4(1), 13-34. Recuperado de http://rsych.com/

Panzini, R. G., \& Bandeira, D. R. (2005). Escala de Coping Religioso-Espiritual (Escala CRE): Elaboração e validação de construto. Psicologia em Estudo, 10(3), 507-516. doi: 10.1590/s1413-73722005000300019

Pargament, K. I. (1997). The psychology of religion and coping: Theory, research and practice. New York: The Guilford Press.

Pargament, K., Feuille, M., \& Burdzy, D. (2011). The Brief RCOPE: Current psychometric status of a short measure of religious coping. Religions, 2(1), 51-76. doi: 10.3390/rel2010051

Pargament, K. I., Koenig, H. G., \& Perez, L. (2000). The many methods of religious coping: Development and initial validation of the RCOPE. Journal of Clinical Psychology, 56(4), 519-543. doi: 10.1002/ (SICI) 1097-4679(200004)56:4<519::AIDJCLP6 $>3.0 . C O ; 2-1$

Pargament, K. I., Koenig, H. G., Tarakeshwar, N., Hahn, J. (2001). Religious struggle as a predictor of mortality among medically ill elderly patients: A two-year longitudinal study. Archives of Internal Medicine, 161(15), 1881-1885. doi: 10.1001/ archinte.161.15.1881

Pargament, K. I., Smith, B. W., Koenig, H. G., \& Perez, L. (1998). Patterns of positive and negative religious coping with major life stressors. Journal for the Scientific Study of Religion, 37(4), 710-724. Recuperado de http://www.jstor.org/stable/1388152

Pargament, K. I., \& Raiya, H. A. (2007). A decade of research on the psychology of religion and coping: Things we assumed and lessons we learned. Psyche and Logos, 28(2), 742-766. Recuperado de https:// tidsskrift.dk/psyke/article/viewFile/8398/6958

Pendleton, S. M., Cavalli, K. S., Pargament, K. I., \& Nasr, S. Z. (2002). Religious/spiritual coping in childhood cystic fibrosis: A qualitative study. Pediatrics, 109(1), e8. doi: 10.1542/peds.109.1.e8

Pew Research Center's Forum on Religion \& Public Life
(2012). The global religious landscape: A report on the size and distribution of the world's major religious groups as of 2010. Recuperado de http:// www.pewforum.org/2012/12/18/global-religiouslandscape-exec/

Quiceno, J., \& Vinaccia, S. (2011). Creencias-prácticas y afrontamiento espiritual-religioso y características sociodemográficas en enfermos crónicos. Psychologia, 5(1), 25-36. Recuperado de http:// www.scielo.org.co/pdf/psych/v5n1/v5n1a03.pdf

Raykov, T., \& Shrout, P. E. (2002). Reliability of scales with general structure: Point and interval estimation using a structural equation modeling approach. Structural Equation Modeling, 9(2), 195-212. doi: 10.1207/ S15328007SEM0902_3

Reyes-Estrada, M., Rivera-Segarra, E., Ramos-Pibernus, A., Rosario-Hernández, E., \& Rivera-Medina, C. (2014). Desarrollo y validación de una escala para medir religiosidad en una muestra de adultos en Puerto Rico. Revista Puertorriqueña de Psicología, 25(2), 226-242. Recuperado de http://www.redalyc. org/articulo.oa?id $=233245622005$

Richards, P. S., \& Bergin, A. E. (2014). Handbook of psychotherapy and religious diversity ( $2^{\mathrm{a}}$ ed.). Washington, DC, US: American Psychological Association. doi: 10.1037/14371-000

Rosenstiel, A. K., \& Keefe, F. J. (1983). The use of coping strategies in chronic low back pain patients: Relationship to patient characteristics and current adjustment. Pain, 17(1), 33-44. doi: 10.1016/03043959(83)90125-2

Satorra, A., \& Bentler, P. M. (2001). A scaled difference chisquare test statistic for moment structure analysis. Psychometrika, 66(4), 507-514. doi: 10.1007/ BF02296192

Scharrón del Río, M. del R. (2010). Supuestos, explicaciones y sistemas de creencias: Ciencia, religión y psicología. Revista Puertorriqueña de Psicología, 21(1), 85-112. Recuperado de http://www.redalyc. org/articulo.oa?id=233218111004

Schumacker, R. E., \& Lomax, R. G. (2010). A beginner's 
guide to structural equation modeling ( $3^{\mathrm{a}}$ ed.).

Mahwah, NJ: Erlbaum.

StataCorp. (2017). Stata: Release 15. Statistical Software. College Station, TX: StataCorp LLC.

The Association of Religion Data Archives (2003). Puerto

Rico public opinion. En Religious Affiliation/ Identifiaction. Recuperado de http://www.thearda. com/internationalData/countries/Country_182_5.asp 